PROCEEDINGS OF THE

AMERICAN MATHEMATICAL SOCIETY

Volume 133, Number 7, Pages 2173-2182

S 0002-9939(05)07793-2

Article electronically published on February 15, 2005

\title{
RELATIONS APPROXIMATED BY CONTINUOUS FUNCTIONS
}

\author{
L'. HOLÁ AND R. A. MCCOY \\ (Communicated by Alan Dow)
}

\begin{abstract}
Let $X$ be a Tychonoff space, let $C(X)$ be the space of all continuous real-valued functions defined on $X$ and let $C L(X \times R)$ be the hyperspace of all nonempty closed subsets of $X \times R$. We prove the following result. Let $X$ be a locally connected, countably paracompact, normal $q$-space without isolated points, and let $F \in C L(X \times R)$. Then $F$ is in the closure of $C(X)$ in $C L(X \times R)$ with the locally finite topology if and only if $F$ is the graph of a cusco map. Some results concerning an approximation in the Vietoris topology are also given.
\end{abstract}

\section{INTRODUCTION}

Let $X$ be a Tychonoff space, let $C(X)$ be the space of all continuous real-valued functions defined on $X$ and let $C L(X \times R)$ be the hyperspace of all nonempty closed subsets of $X \times R$, where $R$ is the space of real numbers. It is known (see $\mathrm{Be} 4$, Ho1, Ho2]) that if $X$ is a locally connected, locally compact metric space without isolated points and $F \in C L(X \times R)$, then $F$ can be approximated by continuous functions in the Hausdorff metric if and only if $F$ is the graph of a cusco map. The fundamental result needed to prove the above theorem is due to Cellina $\mathrm{Ce}$.

In this paper we prove the following analogy for the locally finite topology.

Theorem. Let $X$ be a locally connected, countably paracompact normal q-space without isolated points, and let $F \in C L(X \times R)$. Then the following are equivalent:

(a) $F$ can be approximated by continuous functions in the locally finite topology;

(b) $F$ is the graph of a cusco map.

This theorem is an extension of the result referenced above in the following sense. When $X$ is a metrizable space, the locally finite topology on $C L(X)$ is the supremum of all Hausdorff metric topologies corresponding to compatible metrics on $X$ ([BHPV], [NS]). Furthermore, every locally compact metric space is a countably paracompact normal q-space; where a q-space is a space in which every point has a sequence $\left(U_{n}\right)$ of neighbourhoods such that if $x_{n} \in U_{n}$ for each $n$, then $\left(x_{n}\right)$ has a cluster point. This concept of q-space was introduced in [Mi] and has been useful, among other things, for studying function spaces (see [MN]). The class of q-spaces includes first countable spaces and Čech complete spaces, and hence metric spaces and locally compact spaces.

Received by the editors October 14, 2003 and, in revised form, April 8, 2004.

2000 Mathematics Subject Classification. Primary 54C35, 54B20, 54C08.

Key words and phrases. Set-valued mapping, Vietoris topology, locally finite topology, uppersemicontinuous multifunction, usco map, cusco map. 
There are other theorems like this theorem in the literature; see [Be3] for relations that are approximated in the Hausdorff distance by Baire class one functions, and $\mathrm{Mc}$ for the closure of densely continuous forms in the Vietoris topology. Moreover there is a rich literature concerning an approximation of a multifunction from above by a decreasing sequence of "continuous" multifunctions (see [Hu], [DB], DBM]).

\section{Preliminaries}

We refer to Beer Be1] and Engelking [En for basic notions. If $X$ and $Y$ are nonempty sets, a set-valued mapping or multifunction from $X$ to $Y$ is a mapping that assigns to each element of $X$ a (possibly empty) subset of $Y$. If $T$ is a set-valued mapping from $X$ to $Y$, then its graph is $\{(x, y): y \in T(x)\}$.

If $F$ is a subset of $X \times Y$ and $x \in X$, define $F(x)=\{y:(x, y) \in F\}$. Then we can assign to each subset $F$ of $X \times Y$ a set-valued mapping which takes the value $F(x)$ at each point $x \in X$. Then $F$ is the graph of the set-valued mapping. In our paper we will identify mappings with their graphs.

Let $X$ and $Y$ be topological spaces, and let $T$ be a set-valued mapping from $X$ to $Y$. Then $T$ is a connected map if $T(x)$ is connected for all $x \in X$. Also $T$ is a usc map if $T$ is upper-semicontinuous, and $T$ is a cusc map if it is a connected usc map. In addition, $T$ is a usco map $\mathrm{Ch}$ if $T$ is a usc map such that $T(x)$ is a nonempty compact set for all $x \in X$. Similarly, a cusco map is a cusc map such that $T(x)$ is a nonempty compact set for all $x \in X$.

In the literature, the notation cusco [B] is also used for usco maps with convex values in a topological vector space. Since we are working only with multifunctions with values in $R$, both of these notations coincide in our case.

To describe the hypertopologies with which we will work, we need to introduce the following notation. Let $(X, \tau)$ be a topological space and $C L(X)$ be the hyperspace of all nonempty closed subsets of $X$. For $U \subset X$, define

$$
U^{+}=\{A \in C L(X): A \subset U\} \text { and } U^{-}=\{A \in C L(X): A \cap U \neq \emptyset\} .
$$

If $\mathcal{U}$ is a family of open sets in $X$, define $\mathcal{U}^{-}=\bigcap\left\{U^{-}: U \in \mathcal{U}\right\}$.

A subbase for the Vietoris (resp., locally finite) topology on $C L(X)$ (see [Be1]) are the sets of the form $U^{+}$with $U \in \tau$ and of the form $\mathcal{U}^{-}$with $\mathcal{U} \subset \tau$ finite (resp., locally finite).

Throughout the paper $X$ will be a Hausdorff topological space. We use $C L_{V}(X \times R)$ and $C L_{L F}(X \times R)$ to denote the hyperspace of nonempty closed subsets of $X \times R$ with the Vietoris topology and the locally finite topology, respectively.

\section{Relations APproximated By CONTINUOUS FUnCtions}

This section is concerned with necessary conditions for an element $F \in C L(X \times R)$ to be in the closure of $C(X)$ in $C L_{V}(X \times R)$.

Remark 3.1. It is easy to verify that if $F$ is in the closure of $C(X)$ in $C L_{V}(X \times R)$, then $F(x) \neq \emptyset$ for every $x \in X$, and also that $F$ maps isolated points of $X$ to singletons.

Lemma 3.2. Let $X$ be a locally connected regular space. If $F$ is in the closure of $C(X)$ in $C L_{V}(X \times R)$, then $F$ is the graph of a connected map. 
Proof. Suppose, by way of contradiction, that $F$ is in the closure of $C(X)$ in $C L_{V}(X \times R)$ but $F(x)$ is not connected for some $x$ in $X$. Then there exist $r<s<t$ in $R$ such that $(x, r)$ and $(x, t)$ are in $F$ while $(x, s)$ is not. Let $U$ be a connected open neighbourhood of $x$ in $X$ such that the closed set $\bar{U} \times\{s\}$ in $X \times R$ is disjoint from $F$. Define $W$ to be the complement of this closed set in $X \times R$. Also define $W_{1}=U \times(-\infty, s)$ and $W_{2}=U \times(s, \infty)$. Then $W^{+} \cap W_{1}^{-} \cap W_{2}^{-}$is a neighbourhood of $F$ in $C L_{V}(X \times R)$ and must therefore contain some $f \in C(X)$. It follows that $(-\infty, s)$ and $(s, \infty)$ separate the set $f(U)$, which contradicts the fact that $U$ is connected because $f$ is continuous.

Lemma 3.3. Let $X$ be a locally connected regular space. If $F$ is in the closure of $C(X)$ in $C L_{V}(X \times R)$, then $F$ is the graph of a usc map.

Proof. Suppose, by way of contradiction, that $F$ is in the closure of $C(X)$ in $C L_{V}(X \times R)$, but $F$ is not the graph of a usc map. Then there is an $x \in X$ and a neighbourhood $V$ of $F(x)$ in $R$ such that for every neighbourhood $U$ of $x, F\left(x^{\prime}\right)$ is not contained in $V$ for some $x^{\prime} \in U$. Since $F(x)$ is connected by Lemma 3.2, there is an open interval $V^{\prime}$ containing $F(x)$ such that $\overline{V^{\prime}} \subseteq V$, say $V^{\prime}=(a, b)$ (the case for an infinite interval is similar). Let $U$ be a connected neighbourhood of $x$ such that $\bar{U} \times\{a, b\}$ is disjoint from $F$. So there exists an $x^{\prime} \in U$ such that $F\left(x^{\prime}\right)$ is not contained in $V$. Define $W=X \times R \backslash \bar{U} \times\{a, b\}, W_{1}=U \times V^{\prime}$, and $W_{2}=U \times R \backslash \overline{V^{\prime}}$. Then $F \in W^{+} \cap W_{1}^{-} \cap W_{2}^{-}$, so that there is some $f \in W^{+} \cap W_{1}^{-} \cap W_{2}^{-} \cap C(X)$. But then $f(U)$ is contained in $R \backslash\{a, b\}$ and intersects both $(a, b)$ and $R \backslash[a, b]$, which contradicts the fact that $f(U)$ must be connected.

The following example shows that the assumption of local connectedness in each of Lemmas 3.2 and 3.3 is essential.

Example 3.4. Let $X=Q^{+}$be the space of all nonnegative rational numbers with the usual topology. Let $F \in C L(X \times R)$ be such that $F(x)=\{0,1 / x\}$ if $x \neq 0$ and $F(0)=\{0\}$. Then it is easy to verify that $F$ is in the closure of $C(X)$ in $C L_{V}(X \times R)$, but $F$ is neither the graph of an usc map (it is not uppersemicontinuous at 0 ) nor a connected map.

Lemma 3.5. Let $X$ be a q-space. If $F$ is in the closure of $C(X)$ in $C L_{L F}(X \times R)$, then $F$ is the graph of a compact-valued map.

Proof. Let $x \in X$. Suppose, by way of contradiction, that $F(x)$ were not compact. Let $\left\{y_{n}: n \in N\right\}$ be an unbounded sequence in $F(x)$. Without loss of generality, we can suppose that $\left|y_{n+1}\right|>\max \left\{\left|y_{n}\right|, n\right\}$ for every $n \in N$. Let $\left\{V_{n}: n \in N\right\}$ be a discrete sequence of open bounded intervals such that $y_{n} \in V_{n}$ for each $n \in N$. Let $\left\{U_{n}: n \in N\right\}$ be a sequence of open neighbourhoods of $x$ from the definition of the $q$-space. Let $\mathcal{W}=\left\{U_{n} \times V_{n}: n \in N\right\}$. Because $F \in \mathcal{W}^{-}$, there is an $f \in \mathcal{W}^{-} \cap C(X)$. For every $n \in N$ let $\left(x_{n}, f\left(x_{n}\right)\right) \in U_{n} \times V_{n}$. There is a cluster point $s$ of $\left\{x_{n}: n \in N\right\}$. This contradicts the continuity of $f$ at $s$.

Lemma 3.5 is not true with the Vietoris topology on $C L(X \times R)$, as the next example shows.

Example 3.6. Let $X=[0,1]$ and let $F \in C L(X \times R)$ be defined for all $x$ in $X$ by $F(x)=[1, \infty)$ if $x=0, F(x)=[1,1 / x]$ if $x=1 / n$ for $n \in N$, and $F(x)=\{1 / x\}$ otherwise. Then $F$ is in the closure of $C(X)$ in $C L_{V}(X \times R)$, but $F(0)$ is not compact. 


\section{Approximation of Cusco maps by Continuous functions}

In this section we prove the main theorem, Theorem 4.7.

Lemma 4.1. Let $X$ be a countably paracompact normal space. If $W$ is an open subset of $X \times R$ such that $W(x)$ is a nonempty connected set for all $x$ in $X$, then $W^{+} \cap C(X) \neq \emptyset$ in $C L(X \times R)$.

Proof. Let $\phi: X \times(-\pi / 2, \pi / 2) \rightarrow X \times R$ be the homeomorphism defined by $\phi(x, t)=(x, \tan t)$. Define $g, h: X \rightarrow R$ by $g(x)=\sup \{t \in(-\pi / 2, \pi / 2):(x, t) \in$ $\left.\phi^{-1}(W)\right\}$ and $h(x)=\inf \left\{t \in(-\pi / 2, \pi / 2):(x, t) \in \phi^{-1}(W)\right\}$. To show that $g$ is lower semicontinuous, let $x \in X$ and $\varepsilon>0$. Then there is a $t \in(-\pi / 2, \pi / 2)$ such that $(x, t) \in \phi^{-1}(W)$ and $t>g(x)-\varepsilon$. Because $\phi^{-1}(W) \cap X \times(g(x)-\varepsilon, \pi / 2)$ is a neighbourhood of $(x, t)$ in $X \times(-\pi / 2, \pi / 2)$, there are neighbourhoods $U$ of $x$ and $V$ of $t$ such that $U \times V \subseteq \phi^{-1}(W) \cap X \times(g(x)-\varepsilon, \pi / 2)$. Then for every $x^{\prime} \in U, g\left(x^{\prime}\right)>g(x)-\varepsilon$, so that $g$ is lower semicontinuous. A similar argument shows that $h$ is upper semicontinuous. Now since $X$ is a countably paracompact normal space, by Dowker's theorem ( $[\mathrm{En}, 5.5 .20 \mathrm{a})])$ there is an $f \in C(X)$ such that $h(x)<f(x)<g(x)$ for all $x \in X$. In particular, $f(x) \in(-\pi / 2, \pi / 2)$ for all $x \in X$. Then the composition tan of is well defined and in $C(X)$. To show that tan $\circ$ is in $W^{+}$, suppose, by way of contradiction, that there is an $x \in X$ with $\tan (f(x)) \notin W(x)$. There exist $s, t \in(-\pi / 2, \pi / 2)$ such that $(x, s),(x, t) \in \phi^{-1}(W)$ and $s<f(x)<t$. Then $\tan s, \tan t \in W(x)$ and $\tan s<\tan (f(x))<\tan t$, which contradicts the fact that $W(x)$ is connected.

Lemma 4.2. Let $X$ be a countably paracompact normal space without isolated points. If $W, W_{1}, \ldots, W_{n}$ are open subsets of $X \times R$ such that $W(x)$ is nonempty and connected for all $x \in X$ and such that $W \cap W_{i} \neq \emptyset$ for all $i=1, \ldots, n$, then $W^{+} \cap W_{i}^{-} \cap \ldots \cap W_{n}^{-} \cap C(X) \neq \emptyset$ in $C L(X \times R)$.

Proof. Let $f \in W^{+} \cap C(X)$ from Lemma 4.1. For each $i$, let $\left(x_{i}, t_{i}\right) \in W \cap W_{i}$. Since $X$ has no isolated points, there is no loss of generality in assuming that each $W_{i}=U_{i} \times V_{i}$ where $U_{i}$ is open in $X, V_{i}$ is open in $R, \overline{U_{i}} \times \overline{V_{i}} \subseteq W$, and $\overline{U_{i}} \cap \overline{U_{j}}=\emptyset$ for all $i \neq j$ between 1 and $n$. For each $i, W\left(x_{i}\right)$ is connected, so there is a compact interval $J_{i}$, contained in $W\left(x_{i}\right)$ and containing $t_{i}$ and $f\left(x_{i}\right)$ in its interior. Since $f$ is continuous, by modifying $U_{i}$ if necessary, the $f\left(U_{i}\right)$ may be assumed to be in the interior of $J_{i}$. Now using the normality of $X$ and Tietze's Extension Theorem, the function $f$ can be modified to obtain $g \in C(X)$ so that $g$ agrees with $f$ on $X \backslash\left(U_{1} \cup \ldots \cup U_{n}\right)$ and, for each $i, g\left(U_{i}\right) \subseteq J_{i}$ and $g\left(x_{i}\right)=t_{i}$. Then

$$
g \in W^{+} \cap W_{1}^{-} \cap \ldots \cap W_{n}^{-} \cap C(X) .
$$

Let $G \subset X \times R$. We say that $G$ is locally bounded above (resp., below) provided that for every $x \in X$, there exists a neighbourhood $U$ of $x$ in $X$ and there exists a real number $b$ such that for all $z \in U, G(z) \subseteq(-\infty, b]$ (resp., $G(z) \subseteq[b, \infty)$ ). We say that $G$ is locally bounded if it is both locally bounded below and locally bounded above.

Lemma 4.3. Let $X$ be a countably paracompact space, and let $F \in C L(X \times R)$ be the graph of the cusco map. If $W_{0}$ is an open set in $X \times R$ such that $F \subset W_{0}$, then there exists an open set $W$ in $X \times R$ with $F \subset W \subset W_{0}$, with $W(x)$ connected for every $x \in X$ and with $W$ locally bounded. 
Proof. Let $\left\{\left(a_{n}, b_{n}\right): n \in N\right\}$ be an enumeration of intervals with rational endpoints, and let $x \in X$. The set $\{x\} \times F(x)$ is a compact subset of $W_{0}$, so that there are an open set $U_{x}$ in $X$ and $n \in N$ such that

$$
\{x\} \times F(x) \subset U_{x} \times\left(a_{n}, b_{n}\right) \subset W_{0} \text { and } F(z) \subset\left(a_{n}, b_{n}\right) \text { for every } z \in U_{x} .
$$

For every $n \in N$ define

$$
U_{n}=\bigcup\left\{U_{x}: U_{x} \times\left(a_{n}, b_{n}\right) \subset W_{0} \text { and } F(z) \subset\left(a_{n}, b_{n}\right) \text { for every } z \in U_{x}\right\} .
$$

The countable paracompactness of $X$ implies that there is a locally finite refinement $\mathcal{V}$ of $\left\{U_{n}: n \in N\right\}$. Thus for every $V \in \mathcal{V}$ there is $n(V)$ such that $V \subset U_{n(V)}$. Define the open set $W$ by

$$
W=\bigcup\left\{V \times\left(a_{n(V)}, b_{n(V)}\right): V \in \mathcal{V}\right\} .
$$

To show that $W \subset W_{0}$, let $(x, y) \in W$. Then there is a $V \in \mathcal{V}$ with $(x, y) \in$ $V \times\left(a_{n(V)}, b_{n(V)}\right)$, and thus $x \in U_{n(V)}$. So there is a $z \in X$ with $x \in U_{z}$ and $U_{z} \times\left(a_{n(V)}, b_{n(V)}\right) \subset W_{0}$.

To show that $F \subset W$, let $(z, y) \in F$. Let $V \in \mathcal{V}$ be such that $z \in V$, and let $n(V)$ be such that $V \subset U_{n(V)}$. There is an $x \in X$ with $z \in U_{x}$ and $U_{x} \times\left(a_{n(V)}, b_{n(V)}\right) \subset$ $W_{0}$ and $F(z) \subset\left(a_{n(V)}, b_{n(V)}\right)$. Therefore $(z, y) \in V \times\left(a_{n(V)}, b_{n(V)}\right) \subset W$.

To show that $W(x)$ is connected for every $x \in X$, let $x \in X$. Then $W(x)=$ $\bigcup\left\{\left(a_{n(V)}, b_{n(V)}\right): x \in V\right\}$. Now $\mathcal{V}$ is a locally finite family, so there are only finitely many $V \in \mathcal{V}$ with $x \in V$. For every $V \in \mathcal{V}$ with $x \in V$ we have $F(x) \subset$ $\left(a_{n(V)}, b_{n(V)}\right)$, and thus $W(x)$ is connected.

Finally, to show that $W$ is locally bounded, let $x \in X$. There is a neighbourhood $O_{x}$ of $x$ such that $\mathcal{H}=\left\{V \in \mathcal{V}: O_{x} \cap V \neq \emptyset\right\}$ is finite. Thus for every $z \in O_{x}$ we have $W(z) \subset \bigcup\left\{\left(a_{n(V)}, b_{n(V)}\right): V \in \mathcal{H}\right\}$.

Theorem 4.4. Let $X$ be a countably paracompact normal space without isolated points. If $F \in C L(X \times R)$ is the graph of a cusco map, then $F$ is in the closure of $C(X)$ in $C L_{V}(X \times R)$.

Proof. Let $F \in C L(X \times R)$, and let $W_{0}, W_{1}, \ldots, W_{n}$ be open subsets of $X \times R$ such that

$$
F \in W_{0}^{+} \cap W_{1}^{-} \cap \ldots \cap W_{n}^{-} .
$$

By Lemma 4.3 there is an open set $W$ in $X \times R$ with $F \subset W \subset W_{0}$ and with $W(x)$ connected for every $x \in X$. Thus $W, W_{1}, \ldots, W_{n}$ satisfy the conditions of Lemma 4.2 , so there is an $f \in C(X)$ with

$$
f \in W^{+} \cap W_{1}^{-} \cap \ldots \cap W_{n}^{-} \subset W_{0}^{+} \cap W_{1}^{-} \cap \ldots \cap W_{n}^{-} .
$$

Theorem 4.5. Let $X$ be a locally connected, countably paracompact normal space without isolated points, and let $F \in C L(X \times R)$. Then the following are equivalent:

(a) $F$ is in the closure of $C(X)$ in $C L_{V}(X \times R)$ and $F(x)$ is compact for all $x$ in $X$;

(b) $F$ is the graph of a cusco map.

Proof. The proof that (a) implies (b) follows from Lemmas 3.2 and 3.3, and the proof that (b) implies (a) follows from Theorem 4.4. 
Theorem 4.6. Let $X$ be a countably paracompact normal q-space without isolated points. If $F \in C L(X \times R)$ is the graph of a cusco map, then $F$ is in the closure of $C(X)$ in $C L_{L F}(X \times R)$.

Proof. Let $F \in C L(X \times R)$. Let $W_{0}$ be an open subset of $X \times R$ and let $\mathcal{W}$ be a locally finite family of open subsets of $X \times R$ such that

$$
F \in W_{0}^{+} \cap \mathcal{W}^{-} \text {. }
$$

By Lemma 4.3 there is an open set $W$ in $X \times R$ with $F \subset W \subset W_{0}$, with $W(x)$ connected for every $x \in X$ and with $W$ locally bounded. Without loss of generality, we can suppose that every $G \in \mathcal{W}$ is a subset of $W$ and that $G=U \times V$ with $U$ and $V$ open in $X$ and $R$, respectively.

Let $\mathcal{W}=\left\{U_{\lambda} \times V_{\lambda}: \lambda \in \Lambda\right\}$. Then $\left\{U_{\lambda}: \lambda \in \Lambda\right\}$ is a locally finite family. Let $x \in X$. Fix a sequence $\left\{O_{n}: n \in N\right\}$ of neighbourhoods of $x$ such that each sequence $x_{n} \in O_{n}$ clusters in $X$. Put $L_{n}=\left\{\lambda \in \Lambda: O_{n} \cap U_{\lambda} \neq \emptyset\right\}$. Suppose that all $L_{n}$ are infinite. Then there is an infinite sequence $\left\{\lambda_{n}: n \in N\right\}$ such that $\lambda_{n} \in L_{n}$. Choose $x_{n} \in O_{n} \cap U_{\lambda_{n}}$ and let $y \in X$ be a cluster point of $\left\{x_{n}: n \in N\right\}$. There are a neighbourhood $O$ of $y$ and a compact set $K \subset R$ such that $W(z) \subset K$ for every $z \in O$. Since $\mathcal{W}$ is locally finite, we may suppose that the set $\left\{\lambda \in \Lambda:\left(U_{\lambda} \times V_{\lambda}\right) \cap(O \times K) \neq \emptyset\right\}$ is finite. Let $I$ be the infinite set of all $n \in N$ such that $x_{n} \in O$. Then, for each $n \in I$, we have

$$
\left\{x_{n}\right\} \times V_{\lambda_{n}} \subset U_{\lambda_{n}} \times V_{\lambda_{n}} \subset W .
$$

Thus $V_{\lambda_{n}} \subset W\left(x_{n}\right) \subset K$. It follows that

$$
\left(U_{\lambda_{n}} \times V_{\lambda_{n}}\right) \cap(O \times K) \neq \emptyset
$$

for every $n \in I$, a contradiction. Thus some $L_{n}$ must be finite, which implies that $\left\{U_{\lambda}: \lambda \in \Lambda\right\}$ is locally finite at $x$.

Since $X$ is a regular $T_{1}$ space, by [DHP] there is a discrete family $\left\{G_{i}: i \in I\right\}$ of nonempty open sets such that $|I|=|\Lambda|,\left\{\overline{G_{i}}: i \in I\right\}$ is pairwise disjoint and $\left\{G_{i}: i \in I\right\}^{-} \subset\left\{U_{\lambda}: \lambda \in \Lambda\right\}^{-}$. For every $i \in I$ choose $g_{i} \in G_{i}$. Put $\Lambda(i)=\{\lambda \in$ $\left.\Lambda: g_{i} \in U_{\lambda}\right\}$ and $H_{i}=G_{i} \cap \bigcap\left\{U_{\lambda}: \lambda \in \Lambda(i)\right\}$.

For every $\lambda \in \Lambda(i)$, choose $u_{\lambda} \in H_{i}$ such that, for $\lambda \in \Lambda(i)$, all $u_{\lambda}$ are different. Now for every $\lambda \in \Lambda(i)$, let $L_{\lambda} \subset H_{i}$ be an open set such that $\overline{L_{\lambda}} \subset H_{i}$ and such that $\left\{\overline{L_{\lambda}}: \lambda \in \Lambda(i)\right\}$ is a pairwise disjoint family. Thus the family $\left\{\overline{L_{\lambda}}: \lambda \in \Lambda(i), i \in I\right\}$ is also pairwise disjoint. For each $\lambda \in \Lambda$, choose $y_{\lambda} \in V_{\lambda}$. By Lemma 4.1 there is a continuous function $f$ such that $f \in W^{+}$. For every $\lambda \in \Lambda$, the points $y_{\lambda}$ and $f\left(u_{\lambda}\right)$ belong to the set $W\left(u_{\lambda}\right)$. Now $W\left(u_{\lambda}\right)$ is a connected set, so the closed interval $J_{\lambda}$ from $f\left(u_{\lambda}\right)$ to $y_{\lambda}$ is contained in $W\left(u_{\lambda}\right)$. There are open sets $A_{\lambda}$ and $B_{\lambda}$ in $X$ and $R$, respectively, such that $B_{\lambda}$ is an open interval,

$$
\left\{u_{\lambda}\right\} \times J_{\lambda} \subset A_{\lambda} \times B_{\lambda} \subset \overline{A_{\lambda}} \times \overline{B_{\lambda}} \subset W, A_{\lambda} \subset L_{\lambda} \text { and } f\left(A_{\lambda}\right) \subset B_{\lambda} .
$$

For each $\lambda \in \Lambda$, using the normality of $\overline{A_{\lambda}}$ and Tietze's Extension Theorem, there is a continuous function $g_{\lambda}: \overline{A_{\lambda}} \rightarrow \overline{B_{\lambda}}$ such that $g_{\lambda} \uparrow\left(\overline{A_{\lambda}} \backslash A_{\lambda}\right)=f$ and $g_{\lambda}\left(u_{\lambda}\right)=y_{\lambda}$. Now we define a function $g: X \rightarrow R$ as follows: $g(x)=g_{\lambda}(x)$ if $x \in \bigcup\left\{\overline{A_{\lambda}}: \lambda \in \Lambda\right\}$ and $g(x)=f(x)$ otherwise. It is easy to verify that $g$ is continuous and

$$
g \in W^{+} \cap \mathcal{W}^{-} .
$$


Theorem 4.7. Let $X$ be a locally connected, countably paracompact normal q-space without isolated points, and let $F \in C L(X \times R)$. Then the following are equivalent:

(a) $F$ is in the closure of $C(X)$ in $C L_{L F}(X \times R)$;

(b) $F$ is the graph of a cusco map;

(c) $F \cap C \times R$ is a nonempty continuum for every nonempty continuum $C$ in $X$ and $F \cap C \times R$ is connected for all closed connected subsets $C$ of $X$.

Proof. The proof that (a) implies (b) follows from Lemmas 3.2, 3.3 and 3.5, because the Vietoris topology is weaker than the locally finite topology. The proof that (b) implies (a) follows from Theorem 4.6, and the proof that (b) and (c) are equivalent follows from the results below (Lemmas 4.8, 4.9 and Proposition 5.2).

The equivalence of (b) and (c) is true even under weaker conditions than in Theorem 4.7.

Lemma 4.8. Let $X$ be a Hausdorff topological space and let $F \in C L(X \times R)$ be the graph of a cusco map. Then $F \cap C \times R$ is a continuum for every continuum $C$ in $X$.

Proof. Let $C$ be a nonempty continuum in $X$. Since $\bigcup\{F(x): x \in C\}$ is compact in $R$ (see [Be1]), it is sufficient to prove that $F \cap C \times R$ is connected. Suppose that $F \cap C \times R$ is not connected. Then $F \cap C \times R=A \cup B$ where $A$ and $B$ are disjoint nonempty closed subsets of $X \times R$. For each $x \in C, F(x)$ is connected, so that either $\{x\} \times F(x) \subseteq A$ or $\{x\} \times F(x) \subseteq B$. So if $\pi_{X}: X \times R \rightarrow X$ is the projection map, then $\pi_{X}(A) \cap \pi_{X}(B)=\emptyset$. Since $F \cap C \times R$ is compact, both $\pi_{X}(A)$ and $\pi_{X}(B)$ are closed in $X$. Also $\pi_{X}(A)$ and $\pi_{X}(B)$ are nonempty and their union is $C$, so they form a separation of $C$. This contradicts the fact that $C$ is connected.

Lemma 4.9. Let $X$ be a locally connected, regular space, and let $F \in C L(X \times R)$ be such that $F(x) \neq \emptyset$ for every $x$ in $X$. If $F \cap C \times R$ is connected for every closed connected set $C$ in $X$, then $F$ is the graph of a cusc map.

Proof. Let $F \cap C \times R$ be connected for every closed connected set $C$ in $X$. In particular, $F(x)$ is connected for every $x$ in $X$. Suppose, by way of contradiction, that $F$ is not usc. Then there is a point $x \in X$ and a neighbourhood $V$ of $F(x)$ in $R$ such that for every neighbourhood $U$ of $x$, there is $x_{U} \in U$ and $t_{U} \in F\left(x_{U}\right) \backslash V$. We may suppose, without loss of generality, that $V$ is an open interval and that each $t_{U} \geq b$, where $b=\sup V$.

We now show that there is a neighbourhood $U$ of $x$ such that $U \times[b, b+1] \cap F=$ $\emptyset$. Suppose not. Then for every neighbourhood $U$ of $x$, there is $y_{U} \in U$ and $s_{U} \in F\left(y_{U}\right) \cap[b, b+1]$. Since the net $\left\{y_{U}\right\}$ converges to $x$, the net $\left\{\left(y_{U}, s_{U}\right)\right\}$ must have a cluster point $(x, s)$ for some $s \in[b, b+1]$. But $F$ is closed, so that $(x, s) \in F$, which contradicts $F(x) \subseteq V$.

Therefore, since $X$ is a locally connected regular space, there exists a connected open neighbourhood $W$ of $x$ such that $\bar{W} \times[b, b+1] \cap F=\emptyset$. Note that $t_{W}>b+1$. Define $A=F \cap \bar{W} \times(-\infty, b]$ and $B=F \cap \bar{W} \times[b+1, \infty)$. Then $A$ and $B$ are disjoint closed subsets of $X \times R$ such that $A \cup B=F \cap \bar{W} \times R$. Because $\{x\} \times F(x) \subseteq A$ and $\left\{x_{W}\right\} \times F\left(x_{W}\right) \subseteq B, A \neq \emptyset \neq B$. Hence $F \cap \bar{W} \times R$ is not connected. But $\bar{W}$ is a closed connected set in $X$, which is a contradiction. 


\section{Additional Results CONCERning Vietoris approximation}

In this section we present some further results concerning Vietoris approximation and we give examples of cusc maps that cannot be approximated by continuous functions in the Vietoris topology; so the assumption of compactness of values of $F$ in Theorem 4.5 is essential.

Example 5.1. Let $X=[0,1]$ with the usual topology. Let $F \in C L(X \times R)$ be such that $F(x)=\{1 / x\}$ if $x \neq 0$ and $F(0)=[0, \infty)$. Then $F$ is the graph of a cusc map, but $F$ cannot be approximated by continuous functions in the Vietoris topology because of the following proposition.

Proposition 5.2. Let $X$ be a locally connected, countably paracompact normal space. If $F$ is in the closure of $C(X)$ in $C L_{V}(X \times R)$, then $F \cap C \times R$ is connected for all closed connected subsets $C$ of $X$.

Proof. Suppose, by way of contradiction, that $F$ is in the closure of $C(X)$ in $C L_{V}(X \times R)$ but $F \cap C \times R$ is not connected for some closed connected subset $C$ of $X$. Then $F \cap C \times R$ can be partitioned into nonempty closed sets $F_{1}$ and $F_{2}$; say $\left(x_{1}, t_{1}\right) \in F_{1}$ and $\left(x_{2}, t_{2}\right) \in F_{2}$. From Lemma 3.2, $F\left(x_{1}\right)$ and $F\left(x_{2}\right)$ are connected, and are hence contained in $F_{1}$ and $F_{2}$, respectively. It follows that $\left\{x_{1}\right\} \times R$ is disjoint from $F_{2}$ and $\left\{x_{2}\right\} \times R$ is disjoint from $F_{1}$. Define

$$
F_{1}{ }^{\prime}=F_{1} \cup\left\{x_{1}\right\} \times R \text { and } F_{2}{ }^{\prime}=F_{2} \cup\left\{x_{2}\right\} \times R,
$$

which are disjoint closed subsets of $X \times R$. Since $X \times R$ is normal ([Mc]) there exist disjoint open sets $W_{1}$ and $W_{2}$ in $X \times R$ containing $F_{1}{ }^{\prime}$ and ${F_{2}}^{\prime}$, respectively. Finally, define

$$
W=W_{1} \cup W_{2} \cup(X \backslash C) \times R
$$

Then $W^{+}$is a neighbourhood of $F$ in $C L_{V}(X \times R)$, and thus contains some $f \in C(X)$. It follows that $W_{1}$ and $W_{2}$ separate $\{(x, f(x)): x \in C\}$, so that $\{(x, f(x)): x \in C\}$ is not connected as a subspace of $X \times R$. But $\{(x, f(x)): x \in C\}$ is homeomorphic to $C$ because $f$ is continuous. Since $C$ is connected, this is a contradiction.

Example 5.3. Let $X=[0,1] \times R$, and let $F \in C L(X \times R)$ be defined for all $x=(s, t)$ in $X$ by $F(x)=[1, \infty)$ if $s=0, F(x)=[1,1 / s]$ if $s=1 / n$ for $n \in N$ and $t \notin(n, n+1)$ and $F(x)=\{1 / s\}$ otherwise. Then $F$ is the graph of a cusc map, but $F$ cannot be approximated by continuous functions in the Vietoris topology because of the following proposition.

Proposition 5.4. If $F$ is in the closure of $C(X)$ in $C L_{V}(X \times R)$, then each closed connected set in $X \times R \backslash F$ is locally bounded above or locally bounded below.

Proof. Let $F$ be in the closure of $C(X)$ in $C L_{V}(X \times R)$ and let $G$ be a closed connected set in $X \times R \backslash F$. Define $W=X \times R \backslash G$. Then $F \in W^{+}$, so there is an $f \in W^{+} \cap C(X)$. Let

$$
G_{+}=\{(x, t) \in G: t>f(x)\} \quad \text { and } \quad G_{-}=\{(x, t) \in G: t<f(x)\} .
$$

Since the graph of $f$ is closed in $X \times R$ and disjoint from $G, G_{+}$and $G_{-}$are open subsets of $G$ that partition $G$. But $G$ is connected, so that one of $G_{+}$and $G_{-}$is empty: say $G_{+}=\emptyset$. Then $G=G_{-}$. To show that $G$ is locally bounded above (in this case), let $x \in X$. Define $b=f(x)+1$. By the continuity of $f$, there 
is a neighbourhood $U$ of $x$ such that $f(z)<f(x)+1$ for all $z \in U$, and hence $G(z) \subseteq(-\infty, b)$ for all $z \in U$.

The conditions in this section that are necessary for an element of $C L_{V}(X \times R)$ to be approximated by a continuous function may suggest a solution to the following problem.

Problem 5.5. Find conditions that are both necessary and sufficient for an $F \in$ $C L(X \times R)$ to be in the closure of $C(X)$ in $C L_{V}(X \times R)$.

Remark 5.6. The above problem was solved by the authors for ultraparacompact spaces $X$ in a forthcoming paper.

\section{ACKNOWLEDGMENT}

We would like to thank the referee for suggesting the use of q-spaces in place of locally compact spaces.

\section{REFERENCES}

[Be1] G. Beer, Topologies on closed and closed convex sets, Kluwer Academic Publisher, 1993. MR1269778 (95k:49001)

[Be2] G. Beer, The approximation of real functions in the Hausdorff metric, Houston J. of Math. 10 (1984), 325-338. MF0763235 (85m:41045)

[Be3] G. Beer, On functions that approximate relations, Proc. Amer. Math. Soc. 88 (1983), 643-647. MR 0702292 (84m:54018)

[Be4] G. Beer, On a theorem of Cellina for set valued functions, Rocky Mountain J. of Math. 18 (1988), 37-47. MR0935726 (89c:54034)

[BHPV] G. Beer, J. Himmelberg, K. Prikry and F.S. Van Vleck, The locally finite topology on $2^{X}$, Proc. Amer. Math. Soc. 101 (1987), 168-172. MR0897090|(88f:54014)

[Bo] J.M. Borwein, Minimal CUSCOS and Subgradients of Lipschitz Functions, Fixed point theory and applications (Marseille, 1989), 57-81, Pitman Res. Notes Math. Ser., 252, Longman Sci. Tech., Harlow, 1991. MR.1122818 (92j:46077)

[Ce] A. Cellina, A further result on the approximation of set valued mappings, Rendiconti Acc. Naz. Lincei 48 (1970), 412-416. MR0276935 (43:2675)

[DB] F. De Blasi, Characterizations of certain classes of semicontinuous multifunctions by continuous approximation, J. Math. Anal. Appl. 106 (1985), 1-18. MR0780314 (86h:54021)

[DBM] F. De Blasi and J. Myjak, On continuous approximations for multifunctions, Pacific J. Math. 123 (1986), 9-31. MR0834135 (87g:54047)

[DHP] G. Di Maio, L'. Holá, J. Pelant, Properties related to the first countability of hyperspace topologies, Questions and Answers in General Topology 19 (2001), 139-157. MR1815355 (2002a:54004)

[Ch] J.P.R. Christensen, Theorems of Namioka and R.E. Johnson type for upper semicontinuous and compact valued set-valued mappings, Proc. Amer. Math. Soc. 86 (1982), 649-655. MR.0674099 (83k:54014)

[En] R. Engelking, General Topology, Helderman, Berlin, 1989.

[Ho1] L'. Holá, On relations approximated by continuous functions, Acta Universitatis Carolinae - Mathematica et Physica 28 (1987), 67-72. MR0932741 (89e:54031)

[Ho2] L'. Holá, Hausdorff metric on the space of upper semicontinuous multifunctions, Rocky Mountain J. Math. 22 (1992), 601-610. MR1180723 (93j:54010)

[Hu] M. Hukuhara, Sur l'application semi-continue dont la valeur est un compact convexe, Funkcial. Ekvac. 10 (1967), 43-66. MR0222856 (36:5906)

[Mc] R.A. McCoy, Densely continuous forms in Vietoris hyperspaces, Set-Valued Analysis 8 (2000), 267-271. MF 1790485 (2001h:54017)

[MN] R.A. McCoy and I. Ntantu, Topological Properties of Spaces of Continuous Functions, Springer-Verlag, Berlin, 1988. MR0953314|(90a:54046) 
[Mi] E. Michael, A note on closed maps and compact sets, Isreal J. Math. 2 (1964), 173-176. MR0177396 (31:1659)

[NS] S.A. Naimpally and P.L. Sharma, Fine uniformity and the locally finite hyperspace topology, Proc. Amer. Math. Soc. 103 (1988), 641-646. MF0943098 (89f:54021)

Mathematical Institute, Slovak Academy of Sciences, Štefánikova 49, 81473 BratiSLAVA, SLOVAKIA

E-mail address: hola@mat.savba.sk

Department of Mathematics, Virginia Polytechnic Institute and State University, BLACKSBURG, ViRginia 24061

E-mail address: mccoy@math.vt.edu 